\title{
Classical Analog of Extended Phase Space SUSY and Its Breaking
}

\author{
Gagik Ter-Kazarian \\ Byurakan Astrophysical Observatory, Aragatsotn District, 378433 Byurakan, Armenia \\ Correspondence should be addressed to Gagik Ter-Kazarian; gago_50@yahoo.com
}

Received 21 April 2013; Accepted 12 May 2013

Academic Editors: Z. Qiao and P. Roy

Copyright (C) 2013 Gagik Ter-Kazarian. This is an open access article distributed under the Creative Commons Attribution License, which permits unrestricted use, distribution, and reproduction in any medium, provided the original work is properly cited.

\begin{abstract}
We derive the classical analog of the extended phase space quantum mechanics of the particle with odd degrees of freedom which gives rise to $(N=2)$-realization of supersymmetry (SUSY) algebra. By means of an iterative procedure, we find the approximate ground state solutions to the extended Schrödinger-like equation and use these solutions further to calculate the parameters which measure the breaking of extended SUSY such as the ground state energy. Consequently, we calculate a more practical measure for the SUSY breaking which is the expectation value of an auxiliary field. We analyze nonperturbative mechanism for extended phase space SUSY breaking in the instanton picture and show that this has resulted from tunneling between the classical vacua of the theory. Particular attention is given to the algebraic properties of shape invariance and spectrum generating algebra.
\end{abstract}

\section{Introduction}

An interesting question of keeping the symmetry between canonical coordinates and momenta in the process of quantization deserves an investigation. From its historical development, this aspect of statistical quantum mechanics, unfortunately, has attracted little attention. However, much use has been made of the technique of ordering of canonical coordinates $(q)$ and momenta $(p)$ in quantum mechanics $[1,2]$. It was observed that the concept of an extended Lagrangian $\mathscr{L}(p, q, \dot{p}, \dot{q})$ in phase space allows a subsequent extension of Hamilton's principle to actions minimum along the actual trajectories in $(p, q)$-, rather than in $q$-space. This leads to the phase space formulation of quantum mechanics. Consequently this formalism was developed further in [3] by addressing the extended phase space stochastic quantization of Hamiltonian systems with first class holonomic constraints. This in a natural way results in the FaddeevPopov conventional path-integral measure for gauge systems. Continuing along this line in the present paper we address the classical analog of the extended phase space $(N=2)$ SUSY quantum mechanics [4] of the particles which have both bosonic and fermionic degrees of freedom, that is, the quantum field theory in $(0+1)$-dimensions in $(q, p)$ space, exhibiting supersymmetry (for conventional SUSY quantum mechanics see [5-12]). We analyze in detail the nonperturbative mechanism for supersymmetry breaking in the instanton picture ([13]). This paper has been organized as follows. In the first part (Sections 2 and 3), we derive the classical analog of the extended phase space SUSY quantum mechanics and obtain the integrals of motion. Consequently, we describe the extended phase space $(N=2)$-SUSY algebra. In the second part (Sections 4 and 5), by means of an iterative scheme, first, we find the approximate ground state solutions to the extended Schrödinger-like equation and then calculate the parameters which measure the breaking of extended SUSY such as the ground state energy. We calculate a more practical measure for the SUSY breaking, in particular in field theories, which is the expectation value of an auxiliary field. We analyze nonsperturbative mechanism for extended phase space SUSY breaking in the instanton picture and show that this has resulted from tunneling between the classical vacua of the theory. Section 6 deals with the independent group theoretical methods with nonlinear extensions of Lie algebras from the perspective of extended phase space SUSY quantum mechanics and, further, shows how it can be useful for spectrum generating algebra. The concluding remarks are given in Section 7. Unless otherwise stated we take the geometrized units $(\hbar=c=1)$. Also, an implicit summation on repeated indices is assumed throughout this paper. 


\section{The Integrals of Motion}

For the benefit of those not familiar with the framework of extended phase space quantization, enough details are given in the following to make the rest of the paper understandable. The interested reader is invited to consult the original papers $[1,2]$ for further details. In the framework of the proposed formalism, the extended Lagrangian can be written as

$$
\mathscr{L}_{\text {ext }}(p, q, \dot{p}, \dot{q})=-\dot{q}_{i} p_{i}-q_{i} \dot{p}_{i}+\mathscr{L}^{q}+\mathscr{L}^{p},
$$

where a dynamical system with $N$ degrees of freedom described by the $2 N$ independent coordinates $q=\left(q_{1}, \ldots q_{N}\right)$ and momenta $p=\left(p_{1}, \ldots p_{N}\right)$ which are not, in general, canonical pairs. A Lagrangian $\mathscr{L}^{q}(q, \dot{q})$ is given in $q$-representation and the corresponding $\mathscr{L}^{p}(p, \dot{p})$ in $p$ representation. A dot will indicate differentiation with respect to $t$. The independent nature of $p$ and $q$ gives the freedom of introducing a second set of canonical momenta for both $p$ and $q$ through the extended Lagrangian

$$
\begin{aligned}
& \pi_{q_{i}}=\frac{\partial \mathscr{L}_{\mathrm{ext}}}{\partial \dot{q}_{i}}=\frac{\partial \mathscr{L}^{q}}{\partial \dot{q}_{i}}-p_{i}, \\
& \pi_{p_{i}}=\frac{\partial \mathscr{L}_{\mathrm{ext}}}{\partial \dot{p}_{i}}=\frac{\partial \mathscr{L}^{p}}{\partial \dot{p}_{i}}-q_{i} .
\end{aligned}
$$

One may now define an extended Hamiltonian

$$
\begin{aligned}
H_{\mathrm{ext}}\left(p, q, \pi_{p}, \pi_{q}\right) & =\pi_{q_{i}} \dot{q}_{i}+\pi_{p_{i}} \dot{p}_{i}-\mathscr{L}_{\mathrm{ext}}(p, q, \dot{p}, \dot{q}) \\
& =H\left(p+\pi_{q}, q\right)-H\left(p, q+\pi_{p}\right),
\end{aligned}
$$

where $H(p, q)=p_{i} \dot{q}_{i}-\mathscr{L}^{q}=q_{i} \dot{p}_{i}-\mathscr{L}^{p}$ is the conventional Hamiltonian of the system. In particular, vanishing of $\pi_{q} /$ or $\pi_{p}$ is the condition for $p$ and $q$ to constitute a canonical pair. In the language of statistical quantum mechanics this choice picks up a pure state (actual path). Otherwise, one is dealing with a mixed state (virtual path). One may, however, envisage that the full machinery of the conventional quantum mechanical dynamics is extendible to the extended dynamics as alluded to the above. Here $p$ and $q$ will be considered as independent $c$-number operators on the integrable complex function $\chi(q, p)$. One of the key assumptions of extended phase space quantization $[1,2]$ is the differential operators and commutation brackets for $\pi_{p}$ and $\pi_{q}$ borrowed from the conventional quantum mechanics as follows:

$$
\begin{array}{ll}
\pi_{q_{i}}=-i \frac{\partial}{\partial q_{i}}, & {\left[\pi_{q_{i}}, q_{j}\right]=-i \delta_{i j},} \\
\pi_{p_{i}}=-i \frac{\partial}{\partial p_{i}}, & {\left[\pi_{p_{i}}, p_{j}\right]=-i \delta_{i j} .}
\end{array}
$$

Note also the following:

$$
\begin{aligned}
{\left[p_{i}, q_{j}\right] } & =\left[p_{i}, p_{j}\right]=\left[q_{i}, q_{j}\right]=\left[\pi_{p_{i}}, \pi_{q_{j}}\right] \\
& =\left[\pi_{p_{i}}, \pi_{p_{j}}\right]=\left[\pi_{q_{i}}, \pi_{q_{j}}\right]=0 .
\end{aligned}
$$

By the virtue of (4) and (5), $H_{\text {ext }}$ is now an operator on $\chi$. Along the trajectories in $(p, q)$-space, however, it produces the state functions, $\chi(p, q, t)$, via the following Schrödingerlike equation:

$$
i \frac{\partial}{\partial t} \chi=H_{\text {ext }} \chi
$$

Solutions of (6) are

$$
\begin{aligned}
\chi(q, p, t) & =\bar{\chi}_{r}(q, p, t) e^{-i p q} \\
& =a_{\alpha \beta} \psi_{\alpha}(q, t) \phi_{\beta}^{*}(p, t) e^{-i p q},
\end{aligned}
$$

where $a=a^{\dagger}$, positive definite, $\operatorname{tr} a=1$, and $\psi_{\alpha}$ and $\phi_{\alpha}^{*}$ are solutions of the conventional Schrödinger equation in $q$ - and $p$-representations, respectively. They are mutually Fourier transforms. Note that the $\alpha$ and $\beta$ are not, in general, eigenindices. The normalizable $\chi\left(\int \chi d p d q=\operatorname{tr}(a)=1\right)$ is a physically acceptable solution. The exponential factor is a consequence of the total time derivative, $-d(q p) / d t$, in (1) which can be eliminated. Actually, it is easily verified that

$$
\left(p+\pi_{q}\right) \chi_{r}(q, p, t)=\left(\pi_{q} \bar{\chi}_{r}(q, p, t)\right) e^{-i p q}
$$

and so on. Substitution of (8) in (6) gives

$$
i \frac{\partial}{\partial t} \bar{\chi}(q, p, t)=\bar{H}_{\mathrm{ext}} \bar{\chi}_{r}(q, p, t)
$$

provided by the reduced Hamiltonian, $\bar{H}_{\text {ext }}$. From now on we replace $H_{\text {ext }}$ by $\bar{H}_{\text {ext }}$ and $\chi_{r}(q, p, t)$ by $\bar{\chi}_{r}(q, p, t)$, respectively, and retain former notational conventions.

It is certainly desirable to derive the classical analog of the extended phase space quantum mechanics of the particle with odd degrees of freedom directly from what may be taken as the first principle. Therefore, following [10-12], let us consider a nonrelativistic particle of unit mass with two $(\alpha=1,2)$ odd (Grassmann) degrees of freedom. The classical extended Lagrangian equation (1) can be written

$$
\begin{aligned}
\mathscr{L}_{\text {ext }}(p, q, \dot{p}, \dot{q})= & -\dot{q} p-q \dot{p}+\frac{1}{2} \dot{q}^{2}-F(q)+\frac{1}{2} \dot{p}^{2} \\
& -G(p)-R(q, p) N+\frac{1}{2} \psi_{\alpha} \dot{\psi}_{\alpha},
\end{aligned}
$$

provided by $N=\psi_{1} \psi_{2}=-i \psi_{+} \psi_{-}$. Here $F(q): \mathscr{R} \rightarrow$ $\mathscr{R}, G(p): \mathscr{R} \rightarrow \mathscr{R}$, and $R(q, p): \mathscr{R} \rightarrow \mathscr{R}$ are arbitrary piecewise continuously differentiable functions given over the 1 -dimensional Euclidean space $\mathscr{R}$. The $\psi_{\alpha}$ are two odd (Grassmann) degrees of freedom. The nontrivial PoissonDirac brackets of the system (10) are

$$
\begin{aligned}
& \left\{q, \pi_{q}\right\}=1, \quad\left\{p, \pi_{p}\right\}=1, \quad\left\{\psi_{\alpha}, \psi_{\beta}\right\}=\delta_{\alpha \beta}, \\
& \left\{\psi_{+}, \psi_{-}\right\}=1, \quad \psi_{ \pm}^{2}=0, \quad \psi_{ \pm}=\frac{1}{\sqrt{2}}\left(\psi_{1} \pm i \psi_{2}\right) .
\end{aligned}
$$

The extended Hamiltonian $H_{\text {ext }}$ (3) reads

$$
\begin{aligned}
H_{\text {ext }}\left(p, q, \pi_{p}, \pi_{q}\right)= & \frac{1}{2}\left(p+\pi_{q}\right)^{2}+F^{2}(q)-\frac{1}{2}\left(q+\pi_{p}\right)^{2} \\
& -G^{2}(p)+R(q, p) N
\end{aligned}
$$


which, according to (8), reduces to

$$
\begin{aligned}
H_{\mathrm{ext}}\left(p, q, \pi_{p}, \pi_{q}\right)= & \frac{1}{2} \pi_{q}^{2}+F^{2}(q)-\frac{1}{2} \pi_{p}^{2} \\
& -G^{2}(p)+R(q, p) N .
\end{aligned}
$$

The Hamiltonian equation (13) yields the following equations of motion:

$$
\begin{aligned}
& \dot{q}=\pi_{q}, \quad \dot{p}=\pi_{p}, \quad \dot{\pi}_{q}=-F_{q}^{\prime}(q)-R_{q}^{\prime}(q, p) N \\
& \dot{\pi}_{p}=-G_{p}^{\prime}(p)+R_{p}^{\prime}(q, p) N, \quad \dot{\psi}_{ \pm}= \pm i R(q, p) \psi_{ \pm} .
\end{aligned}
$$

A prime will indicate differentiation with respect either to $q$ or $p$. Thus, $N$ is the integral of motion additional to $H_{\text {ext }}$. Along the trajectories $q(t)$ and $p(t)$ in $(p, q)$-spaces, the solution to equations of motion for odd variables is

$$
\psi_{ \pm}(t)=\psi_{ \pm}\left(t_{0}\right) \exp \left[ \pm i \int_{t_{0}}^{t} R(q(\tau), p(\tau)) d \tau\right] .
$$

Hence the odd quantities

$$
\theta_{ \pm}=\theta_{ \pm}(t) \exp \left[\mp i \int_{t_{0}}^{t} R(q(\tau), p(\tau)) d \tau\right]
$$

are nonlocal in time integrals of motion. In trivial case $R=$ 0 , we have $\dot{\psi}_{ \pm}=0$ and $\theta_{ \pm}=\theta_{ \pm}$. Suppose that the system has even complex conjugate quantities $B_{q, p_{ \pm}},\left(B_{q, p^{+}}\right)^{*}=B_{q, p^{-}}$, whose evolution looks up to the term proportional to $N$ like the evolution of odd variables in (14). Then local odd integrals of motion could be constructed in the form

$$
Q_{q, p \pm}=B_{q, p \mp} \psi_{ \pm} \cdot
$$

Let us introduce the oscillator-like bosonic variables $B_{q, p \pm}$ in $q$ - and $p$-representations as follows:

$$
\begin{array}{ll}
B_{q \mp}: \mathscr{L}^{2}(\mathscr{R}) \longrightarrow \mathscr{L}^{2}(\mathscr{R}), & B_{q_{\mp}}=\left[p+\pi_{q} \pm i W(q)\right], \\
B_{p \mp}: \mathscr{L}^{2}(\mathscr{R}) \longrightarrow \mathscr{L}^{2}(\mathscr{R}), & B_{p \mp}=\left[q+\pi_{p} \pm i V(p)\right] .
\end{array}
$$

In the expressions (18), $W(q): \mathscr{R} \rightarrow \mathscr{R}$ and $V(p): \mathscr{R} \rightarrow \mathscr{R}$ are the piecewise continuously differentiable functions called SUSY potentials. In particular case if $R(q, p)=R_{q}(q)-R_{p}(p)$, for the evolution of $B_{q, p \pm}$ we obtain

$$
\begin{aligned}
& \dot{B}_{q \mp}=\left[-\left(F_{q}^{\prime}+R_{q}^{\prime} N\right) \pm i W_{q}^{\prime}(q)\left(p+\pi_{q}\right)\right], \\
& \dot{B}_{p \mp}=\left[-\left(G_{p}^{\prime}+R_{p}^{\prime} N\right) \pm i V_{p}^{\prime}(p)\left(q+\pi_{p}\right)\right] .
\end{aligned}
$$

Consequently,

$$
\begin{aligned}
& \dot{Q}_{q \pm}= \pm i\left[\left(W_{q}^{\prime}-R_{q \pm}^{\prime}\right) \pm i\left(F_{q}^{\prime}-W W_{q}^{\prime}\right) \psi_{\mp}\right], \\
& \dot{Q}_{p \pm}= \pm i\left[\left(V_{p}^{\prime}-R_{p \pm}^{\prime}\right) \pm i\left(G_{p}^{\prime}-V V_{p}^{\prime}\right) \psi_{\mp}\right] .
\end{aligned}
$$

This shows that either $\dot{Q}_{q \pm}=0$ or $\dot{Q}_{p \pm}=0$ when $W_{q}^{\prime}(q)=$ $R_{q_{ \pm}}^{\prime}(q)$ and $F_{q}^{\prime}=(1 / 2)\left(W^{2}\right)_{q}^{\prime}$ or $V_{p}^{\prime}(p)=R_{p \pm}^{\prime}(p)$ and
$G_{p}^{\prime}=(1 / 2)\left(V^{2}\right)_{p}^{\prime}$, respectively. Therefore, when the functions $R_{q, p}$ and $F(q), G(p)$ are related as

$$
\begin{array}{ll}
R_{q \pm}^{\prime}(q)=W_{q}^{\prime}(q), & F_{q}=\frac{1}{2}\left(W^{2}\right)+C_{q}, \\
R_{p \pm}^{\prime}(p)=V_{p}^{\prime}(p), & F_{p}=\frac{1}{2}\left(V^{2}\right)+C_{p},
\end{array}
$$

where $C_{q, p}$ are constants, then odd quantities $Q_{q, p \pm}$ are integrals of motion in addition to $H_{\text {ext }}$ and $N$. According to (3) and (13), let us present $H_{\text {ext }}$ in the form $H_{\text {ext }}=H_{q}-H_{p}$, where

$$
\begin{aligned}
& H_{q}=\frac{1}{2} \pi_{q}^{2}+F^{2}(q)+R_{q} N \\
& H_{p}=\frac{1}{2} \pi_{p}^{2}+G^{2}(p)+R_{p} N
\end{aligned}
$$

Then, $Q_{q, p \pm}$ and $N$ together with the $H_{q}$ and $H_{p}$ form the classical analog of the extended phase space SUSY algebra as follows:

$$
\begin{aligned}
& \left\{Q_{q, p+}, Q_{q, p^{-}}\right\}=-i\left(H_{q, p}-C_{q, p}\right), \\
& \left\{H_{q, p}, Q_{q, p^{ \pm}}\right\}=\left\{Q_{q, p \pm}, Q_{q, p^{ \pm}}\right\}=0, \\
& \left\{N, Q_{q, p^{ \pm}}\right\}= \pm i Q_{q, p^{ \pm}}, \quad\left\{N, H_{q, p}\right\}=0,
\end{aligned}
$$

with constants $C_{q, p}$ playing a role of a central charges in $(q, p)$ spaces; $N$ is classical analog of the grading operator. Putting $C_{q}=C_{p}=0$, we arrive at the classical analog of the extended phase space SUSY quantum mechanics given by the extended Lagrangian

$$
\begin{aligned}
\mathscr{L}_{\text {ext }}(p, q, \dot{p}, \dot{q})= & \frac{1}{2} \pi_{q}^{2}-\frac{1}{2} W^{2}(q)+\frac{1}{2} \pi_{p}^{2}-\frac{1}{2} V^{2}(p) \\
& +\psi_{1} \psi_{2}\left(W_{q}^{\prime}+V_{p}^{\prime}\right)+\frac{1}{2} \psi_{\alpha} \dot{\psi}_{\alpha} .
\end{aligned}
$$

We conclude that the classical system (10) is characterized by the presence of two additional local in time odd integrals of motion equation (17) being supersymmetry generators. Along the actual trajectories in $q$-space, (24) reproduces the results obtained in [6].

\section{The Path Integral Formulation}

In the matrix formulation of extended phase space $(N=2)$ SUSY quantum mechanics, the $\widehat{\psi}_{ \pm}$will be two real fermionic creation and annihilation nilpotent operators describing the fermionic variables. The $\widehat{\psi}_{ \pm}$, having anticommuting $c$ number eigenvalues, imply

$$
\begin{aligned}
& \widehat{\psi}_{ \pm}=\sqrt{\frac{1}{2}}\left(\widehat{\psi}_{1} \pm i \widehat{\psi}_{2}\right), \quad\left\{\widehat{\psi}_{\alpha}, \widehat{\psi}_{\beta}\right\}=\delta_{\alpha \beta}, \\
& \left\{\widehat{\psi}_{+}, \widehat{\psi}_{-}\right\}=1, \quad \widehat{\psi}_{ \pm}^{2}=0 .
\end{aligned}
$$

They can be represented by finite dimensional matrices $\widehat{\psi}_{ \pm}=$ $\sigma^{ \pm}$, where $\sigma^{ \pm}=\left(\sigma_{1} \pm \sigma_{2}\right) / 2$ are the usual raising and lowering 
operators for the eigenvalues of $\sigma_{3}$ which is the diagonal Pauli matrix. The fermionic operator $\widehat{f}$ reads $\hat{f}: \mathscr{C}^{2} \rightarrow \mathscr{C}^{2}, \widehat{f}=$ $(1 / 2)\left[\widehat{\psi}_{+}, \widehat{\psi}_{-}\right]$, which commutes with the $H_{\text {ext }}$ and is diagonal in this representation with conserved eigenvalues $\pm 1 / 2$. Due to it the wave functions become two-component objects as follows:

$$
\chi(q, p)=\left(\begin{array}{l}
\chi_{+1 / 2}(q, p) \\
\chi_{-1 / 2}(q, p)
\end{array}\right)=\left(\begin{array}{l}
\chi_{1}(q, p) \\
\chi_{2}(q, p)
\end{array}\right)=\left(\begin{array}{l}
\psi_{1}(q) \phi_{1}(p) \\
\psi_{2}(q) \phi_{2}(p)
\end{array}\right),
$$

where the states $\psi_{1,2}(q), \phi_{1,2}(p)$ correspond to fermionic quantum number $f= \pm 1 / 2$, respectively, in $q$ - and $p$-spaces. They belong to Hilbert space $\mathscr{H}=\mathscr{H}_{0} \otimes \mathscr{C}^{2}=\left[\mathscr{L}^{2}(\mathscr{R}) \otimes\right.$ $\left.\mathscr{L}^{2}(\mathscr{R})\right] \otimes \mathscr{C}^{2}$. Hence the Hamiltonian $H_{\text {ext }}$ of extended phase space $(N=2)$-SUSY quantum mechanical system becomes a $2 \times 2$ matrix as follows:

$$
\begin{aligned}
H_{\text {ext }}= & \left(\begin{array}{cc}
H_{+} & 0 \\
0 & H_{-}
\end{array}\right)=\frac{1}{2}\left(\hat{\pi}_{q}^{2}+W^{2}(\widehat{q})+i W_{q}^{\prime}(\widehat{q})\left[\widehat{\psi}_{1}, \widehat{\psi}_{2}\right]\right) \\
& -\frac{1}{2}\left(\widehat{\pi}_{p}^{2}+V^{2}(\widehat{p})+i V_{q}^{\prime}(\widehat{p})\left[\widehat{\psi}_{1}, \widehat{\psi}_{2}\right]\right) .
\end{aligned}
$$

To infer the extended Hamiltonian equation (27) equivalently one may start from the $c$-number extended Lagrangian of extended phase space quantum field theory in $(0+1)$ dimensions in $q$ - and $p$-spaces as follows:

$$
\begin{aligned}
\mathscr{L}_{\text {ext }}(p, q, \dot{p}, \dot{q})= & -\dot{q} p-q \dot{p}+\frac{1}{2}\left[\left(\frac{d q}{d t}\right)^{2}-W^{2}(q)\right] \\
& +f W_{q}^{\prime}(q)+\frac{1}{2}\left[\left(\frac{d p}{d t}\right)^{2}-V^{2}(p)\right] \\
& +f V_{p}^{\prime}(p) .
\end{aligned}
$$

In dealing with abstract space of eigenstates of the conjugate operator $\widehat{\psi}_{ \pm}$which have anticommuting $c$-number eigenvalues, suppose that $|00-\rangle$ is the normalized zero-eigenstate of $\widehat{q}$ and $\widehat{\psi}_{-}$as follows:

$$
\widehat{q}|00-\rangle=0, \quad \widehat{\psi}_{-}|00-\rangle=0 .
$$

The state $|00+\rangle$ is defined by

$$
|00+\rangle=\widehat{\psi}_{+}|00-\rangle=0,
$$

then $\widehat{\psi}_{+}|00+\rangle=0, \widehat{\psi}_{-}|00+\rangle=|00-\rangle$. Taking into account that $\widehat{\psi}_{ \pm}^{\dagger}=\widehat{\psi}_{\mp}$, we get

$$
\langle\mp 00| \widehat{\psi}_{ \pm}=0, \quad\langle\mp 00| \widehat{\psi}_{\mp}=\langle \pm 00| .
$$

Now we may introduce the notation $\alpha, \beta, \ldots$ for the anticommuting eigenvalues of $\widehat{\psi}_{ \pm}$. Consistency requires

$$
\alpha \widehat{\psi}_{ \pm}=-\widehat{\psi}_{ \pm} \alpha, \quad \alpha|00 \pm\rangle= \pm|00 \pm\rangle \alpha .
$$

The eigenstates of $\hat{q}, \widehat{\psi}_{-}$can be constructed as

$$
|q \alpha-\rangle=e^{-i q \widehat{p}^{-\alpha \widehat{\psi}_{+}}}|00-\rangle,
$$

and thus

$$
\widehat{q}|q \alpha-\rangle=q|q \alpha-\rangle, \quad \hat{\psi}_{-}|q \alpha-\rangle=\alpha|q \alpha-\rangle .
$$

Then, the $\widehat{\pi}_{q}$ and $\widehat{\psi}_{+}$eigenstates are obtained by Fourier transformation as follows:

$$
\begin{aligned}
& |q \beta+\rangle=-\int d \alpha e^{\alpha \beta}|q \alpha-\rangle, \\
& \left|\pi_{q} \alpha \pm\right\rangle=-\int d q e^{i q \pi_{q}}|q \alpha \pm\rangle, \\
& |p \beta+\rangle=-\int d \alpha e^{\alpha \beta}|p \alpha-\rangle, \\
& \left|\pi_{p} \alpha \pm\right\rangle=-\int d p e^{i p \pi_{p}}|p \alpha \pm\rangle,
\end{aligned}
$$

which gives

$$
\begin{aligned}
& \widehat{\pi}_{q}\left|\pi_{q} \alpha \pm\right\rangle=\pi_{q}\left|\pi_{q} \alpha \pm\right\rangle, \\
& \widehat{\psi}_{+}\left|\left(q, \pi_{q}\right) \beta+\right\rangle=\beta\left|\left(q, \pi_{q}\right) \beta+\right\rangle, \\
& \widehat{\pi}_{p}\left|\pi_{p} \alpha \pm\right\rangle=\pi_{p}\left|\pi_{p} \alpha \pm\right\rangle, \\
& \widehat{\psi}_{+}\left|\left(p, \pi_{p}\right) \beta+\right\rangle=\beta\left|\left(p, \pi_{p}\right) \beta+\right\rangle .
\end{aligned}
$$

The following completeness relations hold:

$$
\begin{aligned}
& -\int d \alpha d q|q \alpha \pm\rangle\left\langle\mp \alpha^{*} q\right|=1, \\
& -\int d \alpha \frac{d \pi_{q}}{2 \pi}\left|\pi_{q} \alpha \pm\right\rangle\left\langle\mp \alpha^{*} \pi_{q}\right|=1, \\
& -\int d \alpha d p|p \alpha \pm\rangle\left\langle\mp \alpha^{*} p\right|=1, \\
& -\int d \alpha \frac{d \pi_{p}}{2 \pi}\left|\pi_{p} \alpha \pm\right\rangle\left\langle\mp \alpha^{*} \pi_{p}\right|=1 .
\end{aligned}
$$

The time evolution of the state $|t\rangle$ is now given as follows:

$$
\chi_{-}(q \alpha p \beta t)=-\int d \alpha^{\prime} d q^{\prime} d \beta^{\prime} d p^{\prime} K\left(q \alpha p \beta t \mid q^{\prime} \alpha^{\prime} p^{\prime} \beta^{\prime} t^{\prime}\right) .
$$

The kernel reads

$$
\begin{aligned}
& K\left(q \alpha p \beta t \mid q^{\prime} \alpha^{\prime} p^{\prime} \beta^{\prime} t^{\prime}\right) \\
& \quad=\left\langle+q \alpha^{*} p \beta^{*}\left|e^{-i H_{\text {ext }}\left(t-t^{\prime}\right)}\right| q^{\prime} \alpha^{\prime} p^{\prime} \beta^{\prime}\right\rangle,
\end{aligned}
$$

which can be evaluated by the path integral. Actually, an alternative approach to describe the state space and dynamics of the extended phase space quantum system is by the path integral [3], which reads

$$
\mathscr{K}_{f f^{\prime}}\left(q p t \mid q^{\prime} p^{\prime} t^{\prime}\right)=\left\langle q p f\left|e^{-i H_{\text {ext }}\left(t-t^{\prime}\right)}\right| q^{\prime} p^{\prime} f^{\prime}\right\rangle .
$$

In the path integral equation (40) the individual states are characterized by the energy and the fermionic quantum 
number $f$. With the Hamiltonian $H_{\text {ext }}$, the path integral equation (40) is diagonal as follows:

$$
\begin{aligned}
\mathscr{K}_{f f^{\prime}}\left(q p t \mid q^{\prime} p^{\prime} t^{\prime}\right) \\
\quad=\mathscr{K}_{f f^{\prime}}\left(q t \mid q^{\prime} t^{\prime}\right) \mathscr{K}_{f f^{\prime}}\left(p t \mid p^{\prime} t^{\prime}\right) \\
\quad=\delta_{f f^{\prime}} \int_{q^{\prime}}^{q} \mathscr{D} q \int_{p^{\prime}}^{p} \mathscr{D} p \exp \left(i \int_{t^{\prime}}^{t} \mathscr{L}_{\mathrm{ext}}(p, q, \dot{p}, \dot{q}) d t\right) .
\end{aligned}
$$

Knowing the path integral equation (41), it is sufficient to specify the initial wave function $\chi_{f}\left(q^{\prime}, p^{\prime}, t^{\prime}\right)$ to obtain all possible information about the system at any later time $t$, by

$$
\chi_{f}(q, p, t)=\sum_{f^{\prime}} \int d q^{\prime} d p^{\prime} \mathscr{K}_{f f^{\prime}}\left(q p t \mid q^{\prime} p^{\prime} t^{\prime}\right) \chi_{f^{\prime}}\left(q^{\prime}, p^{\prime}, t^{\prime}\right),
$$

with $\chi_{ \pm 1 / 2}(q, p, t)=\chi_{1,2}(q, p, t)$ (26). In terms of anticommuting $c$-number operators $\zeta$ and $\eta$ defining $\psi=$ $\sqrt{1 / 2}\left(\begin{array}{c}\eta+\zeta \\ i(\eta-\zeta)\end{array}\right)$, the path integral equation (41) becomes

$$
\begin{aligned}
\mathscr{K}\left(q \alpha p \beta t \mid q^{\prime} \alpha^{\prime} p^{\prime} \beta^{\prime} t^{\prime}\right)= & \int_{q^{\prime}, \alpha^{\prime}, p^{\prime}, \beta^{\prime}}^{q, \alpha, p, \beta} \mathscr{D} q \mathscr{D} p \mathscr{D} \zeta \mathscr{D} \eta \\
& \times \exp \left(i \int_{t^{\prime}}^{t} \mathscr{L}_{\mathrm{ext}}(p, q, \dot{p}, \dot{q}) d t\right) .
\end{aligned}
$$

The functional integral is taken over all trajectories from $q^{\prime}, \alpha^{\prime}$ to $q, \alpha$ and $p^{\prime}, \beta^{\prime}$ to $p, \beta$ between the times $t^{\prime}$ and $t$.

\section{Solution of the Extended Schrödinger Equation with Small Energy Eigenvalue $\varepsilon$}

Adopting the technique developed in [13], first, we use the iterative scheme to find the approximate ground state solutions to the extended Schrödinger-like equation

$$
H_{\mathrm{ext}} \chi(q, p)=\left(H_{q}-H_{p}\right) \chi(q, p)=\varepsilon \chi(q, p),
$$

with energy $\varepsilon$. We will then use these solutions to calculate the parameters which measure the breaking of extended SUSY such as the ground state energy. The approximation, which went into the derivation of solutions of (44), meets our interest that the ground state energy $\varepsilon$ is supposedly small. As we mentioned above the solutions for nonzero $\varepsilon$ come in pairs of the form

$$
\chi_{\uparrow}(q, p)=\left(\begin{array}{c}
\chi_{1}(q, p) \\
0
\end{array}\right) \quad \text { or } \quad \chi_{\downarrow}(q, p)=\left(\begin{array}{c}
0 \\
\chi_{2}(q, p)
\end{array}\right),
$$

related by supersymmetry, where $\chi_{1,2}(q, p)=\psi_{1,2}(q) \phi_{1,2}(p)$. The state space of the system is defined by all the normalizable solutions of (44) and the individual states are characterized by the energies $\varepsilon_{q}$ and the fermionic quantum number $f$. One of these solutions is acceptable only if $W(q)$ and $V(p)$ become infinite at both $q \rightarrow \pm \infty$ and $p \rightarrow \pm \infty$, respectively, with the same sign. If this condition is not satisfied, neither of the solutions is normalizable, nor they can represent the ground state of the system. Equation (44) yields the following relations between energy eigenstates with fermionic quantum number $\pm 1 / 2$ :

$$
\begin{aligned}
& {\left[\left(\frac{\partial}{\partial q}+W_{q}(q)\right)-\left(\frac{\partial}{\partial p}+V_{p}(p)\right)\right] \psi_{1}(q) \phi_{1}(p)} \\
& =\sqrt{2 \varepsilon_{q}} \psi_{2}(q) \phi_{1}(p)-\sqrt{2 \varepsilon_{p}} \psi_{1}(q) \phi_{2}(p), \\
& {\left[\left(-\frac{\partial}{\partial q}+W_{q}(q)\right)-\left(-\frac{\partial}{\partial p}+V_{p}(p)\right)\right] \psi_{2}(q) \phi_{2}(p)} \\
& =\sqrt{2 \varepsilon_{q}} \psi_{1}(q) \phi_{2}(p)-\sqrt{2 \varepsilon_{p}} \psi_{2}(q) \phi_{1}(p),
\end{aligned}
$$

where $\varepsilon=\varepsilon_{q}-\varepsilon_{p}$ and $\varepsilon_{q}$ and $\varepsilon_{p}$ are the eigenvalues of $H_{q}$ and $H_{p}$, respectively. The technique now is to devise an iterative approximation scheme to solve (46) and (47) by taking a trial wave function for $\chi_{2}(q, p)$, substitute this into the first equation (46), and integrate it to obtain an approximation for $\chi_{1}(q, p)$. This can be used as an ansatz in the second equation (47) to find an improved solution for $\chi_{2}(q, p)$ and so forth. As it was shown in [13], the procedure converges for well-behaved potentials with a judicious choice of initial trial function. If the $W_{q}$ and $V_{p}$ are odd, then

$$
\psi_{1}(-q)=\psi_{2}(q), \quad \phi_{1}(-p)=\phi_{2}(p)
$$

since they satisfy the same eigenvalue equation. It is straightforward then, for example, to obtain

$$
\begin{gathered}
{\left[\left(\frac{\partial}{\partial q}+W_{q}(q)\right)-\left(\frac{\partial}{\partial p}+V_{p}(p)\right)\right] \psi_{1}(q) \phi_{1}(p)} \\
=\sqrt{2 \varepsilon_{q}} \psi_{1}(-q) \phi_{1}(p)-\sqrt{2 \varepsilon_{p}} \psi_{1}(q) \phi_{1}(-p) .
\end{gathered}
$$

The independent nature of $q$ and $p$ gives the freedom of taking $q=0, p=0$ which yield an expression for energies as follows:

$$
\begin{aligned}
& \sqrt{2 \varepsilon_{q}}=W(0)+\frac{\psi_{1}^{\prime}(0)}{\psi_{1}(0)}, \\
& \sqrt{2 \varepsilon_{p}}=V(0)+\frac{\phi_{1}^{\prime}(0)}{\phi_{1}(0)} .
\end{aligned}
$$

Suppose that the potentials $W_{q}(q)$ and $V_{p}(p)$ have a maximum, at $q_{-}$and $p_{-}$, and minimum, at $q_{+}$and $p_{+}$, respectively. For the simplicity sake we choose the trial wave functions as

$$
\psi_{1,2}^{(0)}(q)=\delta\left(q-q_{ \pm}\right), \quad \phi_{1,2}^{(0)}(p)=\delta\left(p-p_{ \pm}\right)
$$


After one iteration, we obtain

$$
\begin{aligned}
\chi_{1}^{(1)}(q, p)= & \frac{1}{N_{q} N_{p}} \theta\left(q-q_{-}\right) \theta\left(p-p_{-}\right) \\
& \times e^{-\int_{0}^{q} d q^{\prime} W\left(q^{\prime}\right)+\int_{0}^{p} d p^{\prime} V\left(p^{\prime}\right)}, \\
\chi_{2}^{(1)}(q, p)= & \frac{1}{N_{q} N_{p}} \theta\left(q_{+}-q\right) \theta\left(p_{+}-p\right) \\
& \times e^{\int_{0}^{q} d q^{\prime} W\left(q^{\prime}\right)-\int_{0}^{p} d p^{\prime} V\left(p^{\prime}\right)},
\end{aligned}
$$

where $N_{q}$ and $N_{p}$ are the normalization factors. The next approximation leads to

$$
\begin{aligned}
\chi_{1}^{(2)}(q, p)= & \frac{1}{N^{\prime}} e^{-\int_{0}^{q} d q^{\prime} W\left(q^{\prime}\right)+\int_{0}^{p} d p^{\prime} V\left(p^{\prime}\right)} \\
& \times \int_{\max \left(-q, q_{-}\right)}^{\infty} e^{-2 \int_{0}^{q^{\prime}} d q^{\prime \prime} W\left(q^{\prime \prime}\right)} d q^{\prime} \\
& \times \int_{\max \left(-p, p_{-}\right)}^{\infty} e^{2 \int_{0}^{p^{\prime}} d p^{\prime \prime} V\left(p^{\prime \prime}\right)} d p^{\prime}, \\
\chi_{2}^{(2)}(q, p)= & \frac{1}{N^{\prime}} e^{\int_{0}^{q} d q^{\prime} W\left(q^{\prime}\right)-\int_{0}^{p} d p^{\prime} V\left(p^{\prime}\right)} \\
& \times \int_{\infty}^{\min \left(-q, q_{+}\right)} e^{2 \int_{0}^{q^{\prime}} d q^{\prime \prime} W\left(q^{\prime \prime}\right)} d q^{\prime} \\
& \times \int_{\infty}^{\min \left(-p, p_{+}\right)} e^{-2 \int_{0}^{p^{\prime}} d p^{\prime \prime} V\left(p^{\prime \prime}\right)} d p^{\prime} .
\end{aligned}
$$

It can be easily verified that to this level of precision (52) is self-consistent solution. Actually, for example, for $q^{\prime}>q_{-}$the exponential $e^{-2 \int_{0}^{q^{\prime}} W\left(q^{\prime \prime}\right) d q^{\prime \prime}}$ will peak sharply around $q_{+}$and may be approximated by a $\delta$-function $c \delta\left(q_{+}-q^{\prime}\right)$; similarly $e^{2 \int_{0}^{q^{\prime}} W\left(q^{\prime \prime}\right) d q^{\prime \prime}}$ may be replaced approximately by $c \delta\left(q_{-}-q^{\prime}\right)$ for $q^{\prime}<q_{+}$. The same arguments hold for the $p$-space. With these approximations equations (53) reduce to (52). The normalization constant $N^{\prime}$ is

$$
N^{\prime}=\left(\int_{q_{-}}^{\infty} d q e^{-2 \int_{0}^{q} W\left(q^{\prime}\right) d q^{\prime}} \int_{p_{-}}^{\infty} d p e^{-2 \int_{0}^{p^{\prime}} V\left(p^{\prime}\right) d p^{\prime}}\right)^{3 / 2} .
$$

The energy expectation value

$$
\varepsilon=\left(\chi_{1}, H_{\mathrm{ext}} \chi_{1}\right)
$$

gives the same result as that obtained for odd potentials by means of (50) and (53). Assuming the exponentials $e^{-2 \int_{q_{-}}^{0} W(q) d q}$ and $e^{-2 \int_{p_{-}}^{0} V(p) d p}$ to be small, which is correct to the same approximations underlying (55), the difference is negligible and the integrals in both cases may be replaced by Gaussians around $q_{+}$and $p_{+}$, respectively. Hence, it is straightforward to obtain

$$
\varepsilon=\frac{\hbar W^{\prime}\left(q_{+}\right)}{2 \pi} e^{-2 \Delta W / \hbar}-\frac{\hbar V^{\prime}\left(p_{+}\right)}{2 \pi} e^{-2 \Delta V / \hbar},
$$

which gives direct evidence for the SUSY breaking in the extended phase space quantum mechanical system. Here we have reinstated $\hbar$, to show the order of adopted approximation, and its non-perturbative nature. We also denoted

$$
\Delta W=\int_{q_{+}}^{q_{-}} W(q) d q, \quad \Delta V=\int_{p_{+}}^{p_{-}} V(p) d p .
$$

However, a more practical measure for the SUSY breaking, in particular, in field theories is the expectation value of an auxiliary field, which can be replaced by its equation of motion right from the start as follows:

$$
\langle F\rangle=\left(\chi_{\uparrow}, i\left\{Q_{+}, \sigma_{-}\right\} \chi_{\uparrow}\right)
$$

Taking into account the relation $Q_{+} \chi_{\uparrow}=0$, with $Q_{+}$ commuting with $H_{\text {ext }}$, which means that the intermediate state must have the same energy as $\chi,(58)$ can be written in terms of a complete set of states as

$$
\langle F\rangle=i\left(\chi_{\uparrow}, Q_{+} \chi_{\downarrow}\right)\left(\chi_{\downarrow}, \sigma_{-}, \chi_{\uparrow}\right)
$$

According to (55), we have

$$
\begin{aligned}
\varepsilon= & \left\langle H_{\text {ext }}\right\rangle=\left(\chi_{\uparrow}, H_{\text {ext }} \chi_{\uparrow}\right)=\frac{1}{2}\left(\chi_{\uparrow}, Q_{+} \chi_{\downarrow}\right)\left(\chi_{\downarrow}, Q_{-} \chi_{\uparrow}\right) \\
= & \varepsilon_{q}-\varepsilon_{p}=\left\langle H_{q}\right\rangle-\left\langle H_{p}\right\rangle=\left(\psi_{\uparrow}, H_{q} \psi_{\uparrow}\right)-\left(\phi_{\uparrow}, H_{p} \phi_{\uparrow}\right) \\
= & \frac{1}{2}\left(\psi_{\uparrow}, Q_{q+} \psi_{\downarrow}\right)\left(\psi_{\downarrow}, Q_{q-} \psi_{\uparrow}\right) \\
& -\frac{1}{2}\left(\phi_{\uparrow}, Q_{p+} \phi_{\downarrow}\right)\left(\phi_{\downarrow}, Q_{p-} \phi_{\uparrow}\right),
\end{aligned}
$$

where $\chi_{\uparrow \downarrow}=\psi_{\uparrow \downarrow} \phi_{\uparrow \downarrow}, \psi_{\uparrow}=\left(\begin{array}{c}\psi_{1} \\ 0\end{array}\right), \psi_{\downarrow}=\left(\begin{array}{c}0 \\ \psi_{2}\end{array}\right), \phi_{\uparrow}=\left(\begin{array}{c}\phi_{1} \\ 0\end{array}\right)$, and $\phi_{\downarrow}=\left(\begin{array}{c}0 \\ \phi_{2}\end{array}\right)$. From SUSY algebra it follows immediately that

$$
\begin{aligned}
& \frac{1}{\sqrt{\varepsilon}} Q_{-} \chi_{\uparrow}=\chi_{\downarrow}=\widehat{\psi}_{-} \chi_{\uparrow}, \\
& \frac{1}{\sqrt{\varepsilon_{q}}} Q_{q^{-}} \psi_{\uparrow}=\psi_{\downarrow}=\widehat{\psi}_{-} \psi_{\uparrow}, \\
& \frac{1}{\sqrt{\varepsilon_{p}}} Q_{p^{-}} \phi_{\uparrow}=\phi_{\downarrow}=\widehat{\psi}_{-} \phi_{\uparrow} .
\end{aligned}
$$

By virtue of (61), (60) reads

$$
\begin{aligned}
\sqrt{\varepsilon} & \left(\chi_{\uparrow}, Q_{+} \chi_{\downarrow}\right)\left(\chi_{\downarrow}, \widehat{\psi}_{-} \chi_{\uparrow}\right) \\
= & \sqrt{\varepsilon_{q}}\left(\psi_{\uparrow}, Q_{q+} \psi_{\downarrow}\right)\left(\psi_{\downarrow}, \widehat{\psi}_{-} \psi_{\uparrow}\right) \\
& -\sqrt{\varepsilon_{p}}\left(\phi_{\uparrow}, Q_{p+} \phi_{\downarrow}\right)\left(\phi_{\downarrow}, \widehat{\psi}_{-} \phi_{\uparrow}\right),
\end{aligned}
$$


Using the matrix representations of $Q_{q_{+}}, Q_{p+}$, and $\widehat{\psi}_{-}$and the wave functions equation (53), one gets [13]

$$
\begin{aligned}
& \left(\psi_{\downarrow}, \widehat{\psi}_{-} \psi_{\uparrow}\right)=\sqrt{\frac{W^{\prime}\left(q_{+}\right)}{\pi}} e^{-\Delta W} \Delta q, \\
& \left(\phi_{\downarrow}, \widehat{\psi}_{-} \phi_{\uparrow}\right)=\sqrt{\frac{V^{\prime}\left(p_{+}\right)}{\pi}} e^{-\Delta V} \Delta p, \\
& \left(\psi_{\uparrow}, Q_{q_{+}} \psi_{\downarrow}\right)=i \sqrt{\frac{W^{\prime}\left(q_{-}\right)}{\pi}} e^{-\Delta W}, \\
& \left(\phi_{\uparrow}, Q_{p_{+}} \phi_{\downarrow}\right)=i \sqrt{\frac{V^{\prime}\left(p_{-}\right)}{\pi}} e^{-\Delta V},
\end{aligned}
$$

where $\Delta q=q_{+}-q_{-}$and $\Delta p=p_{+}-p_{-}$. Hence

$$
\begin{aligned}
\left(\chi_{\uparrow}, Q_{+} \chi_{\downarrow}\right)\left(\chi_{\downarrow}, \widehat{\psi}_{-} \chi_{\uparrow}\right) & \\
=2 i \sqrt{\varepsilon}[ & \left(\frac{\varepsilon_{q}}{\varepsilon}\right) \sqrt{\varepsilon}_{q} \sqrt{\frac{W^{\prime}\left(q_{-}\right)}{\pi} \Delta q} \\
& -\left(\frac{\varepsilon_{p}}{\varepsilon}\right) \sqrt{\varepsilon_{p}} \sqrt{\left.\frac{V^{\prime}\left(p_{-}\right)}{\pi} \Delta p\right],} \\
\langle F\rangle=-2 \sqrt{\varepsilon} & {\left[\left(\frac{\varepsilon_{q}}{\varepsilon}\right) \sqrt{\frac{W^{\prime}\left(q_{+}\right)}{\pi}} e^{-2 \Delta W} \Delta q\right.} \\
& \left.-\left(\frac{\varepsilon_{p}}{\varepsilon}\right) \sqrt{\frac{V^{\prime}(p)}{\pi}} e^{-2 \Delta V} \Delta p\right] .
\end{aligned}
$$

Along the actual trajectories in $q$-space, (65) reproduces the results obtained in [13].

\section{An Extended SUSY Breaking in the Instanton Picture}

In this Section our goal is to show that the expressions equations (64) and (65) can be obtained in the path integral formulation of the theory by calculating the matrix elements, that is, the effect of tunneling between two classical vacua by using a one-instanton background. That is, the matrix elements of $\widehat{\psi}_{ \pm}, Q_{q \pm}$, and $Q_{p \pm}$ can be calculated in the background of the classical solution $\dot{q}_{c}=-W_{c}$ and $\dot{p}_{c}=-V_{c}$. In doing this we rewrite the matrix element equation (62) in terms of eigenstates of the conjugate operator $\widehat{\psi}_{ \pm}$as follows:

$$
\begin{aligned}
& \sqrt{\varepsilon}\left\langle\left\langle 0 q_{+} p_{+}\left|e^{-i H_{\mathrm{ext}}(T-t)} Q_{+} e^{-i H_{\mathrm{ext}}(T+t)}\right| q_{-} p_{-} 0-\right\rangle\left\langle-0 q_{-} p_{-}\right|\right. \\
&\left.\times e^{-i H_{\mathrm{ext}}(T-t)} \widehat{\psi}_{-} e^{-i H_{\mathrm{ext}}(T+t)}\left|q_{+} p_{+} 0+\right\rangle\right]_{T \rightarrow-i \infty} \\
&=\sqrt{\varepsilon_{q}}\left[\left\langle+0 q_{+}\left|e^{-i H_{q}(T-t)} Q_{q_{+}} e^{-i H_{q}(T+t)}\right| q_{-} 0-\right\rangle\left\langle-0 q_{-}\right|\right. \\
&\left.\times e^{-i H_{q}(T-t)} \widehat{\psi}_{-}\left|e^{-i H_{q}(T+t)} q_{+} 0+\right\rangle\right]_{T \rightarrow-i \infty}
\end{aligned}
$$

$$
\begin{aligned}
-\sqrt{\varepsilon_{p}} & {\left[\left\langle+0 p_{+}\right| e^{-i H_{p}(T-t)} Q_{p_{+}} e^{-i H_{p}(T+t)}\right.} \\
& \left.\times\left|p_{-} 0-\right\rangle\left\langle-0 p_{-}\left|\widehat{\psi}_{-}\right| p_{+} 0+\right\rangle\right]_{T \rightarrow-i \infty},
\end{aligned}
$$

in the limit $T \rightarrow-i \infty$. This reduces to

$$
\begin{aligned}
\sqrt{\varepsilon}\langle & \left.+0 q_{+} p_{+}\left|Q_{+}\right| q_{-} p_{-} 0-\right\rangle\left\langle-0 q_{-} p_{-}\left|\widehat{\psi}_{-}\right| q_{+} p_{+} 0+\right\rangle \\
= & \sqrt{\varepsilon_{q}}\left\langle+0 q_{+}\left|Q_{q_{+}}\right| q_{-} 0-\right\rangle\left\langle-0 q_{-}\left|\widehat{\psi}_{-}\right| q_{+} 0+\right\rangle \\
& -\sqrt{\varepsilon_{p}}\left\langle+0 p_{+}\left|Q_{p_{+}}\right| p_{-} 0-\right\rangle\left\langle-0 p_{-}\left|\widehat{\psi}_{-}\right| p_{+} 0+\right\rangle,
\end{aligned}
$$

which, in turn, can be presented by path integrals defined in terms of anticommuting $c$-number operators $\zeta$ and $\eta$ with Euclidean actions of the instantons in $q$ - and $p$-spaces, respectively. Following [13], these functional integrals include an integration over instanton time $\tau_{0}$ which is due to the problem of zero modes of the bilinear terms in Euclidean actions. This arises from time-transformation of instantons and SUSY transformations on them, respectively. The existence of zero modes gives rise to non-Gaussian behaviour of the functional integral. Due to it the matrix elements above do not receive any contributions from either no-instanton or anti-instanton configurations. The zero mode problem is solved by introducing a collective coordinate $\tau_{0}$ replacing the bosonic zero mode [14]. Whereas; the functional integrals depend only on the difference $\tau-\tau_{0}$. Note also that multiinstanton configurations could contribute in principle, provided they have not more than one normalizable fermionic zero mode. But as it was shown in [13], their contribution is clearly smaller with respect to $\sqrt{2 \varepsilon_{q}}$ and $\sqrt{2 \varepsilon_{p}}$. In the case when the SUSY potentials in $q$ - and $p$-spaces have more than two extrema $q_{\nu}$ and $p_{\mu}, \nu, \mu=1,2, \ldots, N$, one can put conditions on the SUSY potentials as follows

$$
\begin{aligned}
& \int_{0}^{\infty} W\left(q^{\prime}\right) d q^{\prime} \longrightarrow \infty \quad \text { at } q \longrightarrow \pm \infty \text { for } \psi_{0}^{+}, \\
& \int_{0}^{\infty} W\left(q^{\prime}\right) d q^{\prime} \longrightarrow-\infty \text { at } q \longrightarrow \pm \infty \text { for } \psi_{0}^{-},
\end{aligned}
$$

and similar to $V(p)$, that the extrema are well separated as follows: $\int_{q_{\nu}}^{q_{\nu}+1} W\left(q^{\prime}\right) d q^{\prime} \gg 1, \int_{p_{\mu}}^{q_{\mu}+1} V\left(p^{\prime}\right) d p^{\prime} \gg 1$. Around each of the classical minima $q_{\nu}$ and $p_{\mu}$ of the potentials $W^{2}(q)$ and $V^{2}(p)$, respectively, one can approximate the theory by a supersymmetric harmonic oscillator. Then there are $N$ ground states which have zero energy. These states are described by upper or lower component of the wave function, depending on whether $\nu$ and $\mu$ are odd or even. With this provision the functional integrals are calculated in [13], which allow us consequently to write

$$
\begin{aligned}
& \left\langle+0 q_{+}\left|Q_{q_{+}}\right| q_{-} 0-\right\rangle=i \sqrt{\frac{W_{c}^{\prime}\left(q_{+}\right)}{\pi}} e^{-\Delta W_{c},} \\
& \left\langle-0 q_{-}\left|\widehat{\psi}_{-}\right| q_{+} 0+\right\rangle=\sqrt{\frac{W_{c}^{\prime}\left(q_{+}\right)}{\pi}} e^{-\Delta W_{c}} \Delta q_{c},
\end{aligned}
$$


and so forth. Inserting this in (67), we arrive at (64)

$$
\begin{aligned}
\left\langle+0 q_{+} p_{+}\left|Q_{+}\right| q_{-} p_{-} 0-\right\rangle\left\langle-0 q_{-} p_{-}\left|\widehat{\psi}_{-}\right| q_{+} p_{+} 0+\right\rangle \\
=2 i \sqrt{\varepsilon}\left[\left(\frac{\varepsilon_{q}}{\varepsilon}\right) \sqrt{\varepsilon_{q}} \sqrt{\frac{W_{c}^{\prime}\left(q_{+}\right)}{\pi} \Delta q_{c}}\right. \\
-\left(\frac{\varepsilon_{p}}{\varepsilon}\right) \sqrt{\varepsilon_{p}} \sqrt{\left.\frac{V_{c}^{\prime}\left(p_{+}\right)}{\pi} \Delta p_{c}\right],}
\end{aligned}
$$

and, thus, of (65) as its inevitable corollary. This proves that the extended SUSY breaking has resulted from tunneling between the classical vacua of the theory. The corrections to this picture are due to higher-order terms and quantumtunneling effects.

\section{Spectrum Generating Algebra}

An extended Hamiltonian $H_{\text {ext }}$ equation (27) can be treated as a set of two ordinary two-dimensional partner Hamiltonians [4] as follows:

$$
H_{ \pm}=\frac{1}{2}\left[\pi_{q}^{2}-\pi_{p}^{2}+U_{ \pm}(q, p)\right]
$$

provided by partner potentials

$$
\begin{aligned}
& U_{ \pm}(q, p)=U_{q \pm}(q)-U_{p \pm}(p), \\
& U_{q \pm}(q)=W^{2}\left(q, a_{q}\right) \mp W_{q}^{\prime}\left(q, a_{q}\right), \\
& U_{p \pm}(p)=V^{2}\left(p, a_{p}\right) \mp V_{p}^{\prime}\left(p, a_{p}\right) .
\end{aligned}
$$

A subset of the SUSY potentials for which the Schrödingerlike equations are exactly solvable share an integrability conditions of shape-invariance [15] as follows:

$$
U_{+}\left(a_{0}, q, p\right)=U_{-}\left(a_{1}, q, p\right)+R\left(a_{0}\right), \quad a_{1}=f\left(a_{0}\right),
$$

where $a_{0}$ and $a_{1}$ are a set of parameters that specify phasespace-independent properties of the potentials, and the reminder $R\left(a_{0}\right)$ is independent of $(q, p)$.

6.1. Algebraic Properties of Shape Invariance. Using the standard technique, we may construct a series of Hamiltonians $H_{N}, N=0,1,2, \ldots$,

$$
H_{N}=\frac{1}{2}\left[\pi_{q}^{2}-\pi_{p}^{2}+U_{-}\left(a_{N}, q, p\right)+\sum_{k=1}^{N} R\left(a_{k}\right)\right],
$$

where $a_{N}=f^{(N)}(a)$ ( $N$ is the number of iterations). From (72) and (73) we obtain then $N=n+m$ coupled nonlinear differential equations which are the two recurrence relations of Riccati-type differential equations as follows:

$$
\begin{aligned}
& W_{n+1}^{2}(q)+W_{q(n+1)}^{\prime}(q)=W_{n}^{2}(q)-W_{q n}^{\prime}(q)-\mu_{n}, \\
& V_{m+1}^{2}(p)+V_{p(m+1)}^{\prime}(p)=V_{m}^{2}(p)-V_{p m}^{\prime}(p)-v_{m},
\end{aligned}
$$

where we denote $W_{n}(q) \equiv W\left(q, a_{q n}\right), V_{m}(p) \equiv V\left(p, a_{p m}\right)$, $\mu_{n} \equiv R_{q}\left(a_{q n}\right)$, and $\nu_{m} \equiv R_{p}\left(a_{p m}\right)$. Here we admit that for unbroken SUSY, the eigenstates of the potentials $U_{q, p}$, respectively, are

$$
E_{q 0}^{(-)}=0, \quad E_{q n}^{(-)}=\sum_{i=0}^{n-1} \mu_{i}, \quad E_{p 0}^{(-)}=0, \quad E_{p m}^{(-)}=\sum_{j=0}^{m-1} v_{j},
$$

that is, the ground states are at zero energies, characteristic of unbroken supersymmetry. The differential equations (75) can be investigated to find exactly solvable potentials. The shape invariance condition equation (73) can be expressed in terms of bosonic operators as

$$
\begin{aligned}
& B_{+}\left(x, a_{0}\right) B_{-}\left(x, a_{0}\right)-B_{-}\left(x, a_{1}\right) B_{+}\left(x, a_{1}\right) \\
& \quad=R\left(a_{0}\right)=\mu_{0}-v_{0}, \\
& B_{æ+}\left(æ, a_{æ 0}\right) B_{æ-}\left(æ, a_{æ 0}\right)-B_{æ-}\left(æ, a_{æ 1}\right) B_{æ+}\left(æ, a_{æ 1}\right) \\
& \quad=\left(\mu_{0} \text { or } v_{0}\right),
\end{aligned}
$$

where $x(q, p)$ - is the coordinate in $(q, p)$-space $æ$ denotes concisely either $q$ - or $p$-representations (no summation on $æ$ is assumed). To classify algebras associated with the shape invariance, following [16], we introduce an auxiliary variables $\phi\left(\phi_{q}, \phi_{p}\right)$ and define the following creation and annihilation operators:

$$
J_{+}=e^{i k \phi} B_{+}\left(x, \chi\left(i \partial_{\phi}\right)\right), \quad J_{-}=B_{-}\left(x, \chi\left(i \partial_{\phi}\right)\right) e^{-i k \phi},
$$

where $k\left(k_{q}, k_{p}\right)$ are an arbitrary real constants and $\chi\left(\chi_{q}, \chi_{p}\right)$ are an arbitrary real functions. Consequently, the creation and annihilation operators in $(q, p)$-spaces can be written as

$$
\begin{aligned}
& J_{\mathfrak{x}+}=e^{i k_{\mathfrak{x}} \phi_{\mathfrak{x}}} B_{\mathfrak{x}}\left(\mathfrak{x}, \chi_{\mathfrak{x}}\left(i \partial_{\phi_{\mathfrak{x}}}\right)\right), \\
& J_{\mathfrak{x}-}=B_{\mathfrak{x}}\left(\mathfrak{x}, \chi_{\mathfrak{x}}\left(i \partial_{\phi_{\mathfrak{x}}}\right)\right) e^{-i k_{\mathfrak{x}} \phi_{\mathfrak{x}}} .
\end{aligned}
$$

The operators $B_{ \pm}\left(x, \chi\left(i \partial_{\phi}\right)\right)$ and $B_{æ \pm}\left(æ, \chi_{\mathfrak{x}}\left(i \partial_{\phi_{\mathfrak{x}}}\right)\right)$ are the generalization of $(77)$, where $a_{0} \rightarrow \chi\left(i \partial_{\phi}\right)$ and $a_{æ 0} \rightarrow$ $\chi_{\mathfrak{x}}\left(i \partial_{\phi_{\mathfrak{x}}}\right)$. One can easily prove the following relations:

$$
\begin{aligned}
& e^{i k \phi} B_{+}\left(x, \chi\left(i \partial_{\phi}\right)\right)=B_{+}\left(x, \chi\left(i \partial_{\phi}+k\right)\right) e^{i k \phi}, \\
& B_{-}\left(x, \chi\left(i \partial_{\phi}\right)\right) e^{-i k \phi}=e^{-i k \phi} B_{-}\left(x, \chi\left(i \partial_{\phi}+k\right)\right),
\end{aligned}
$$

and that

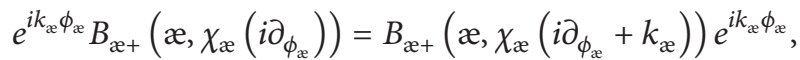

$$
\begin{aligned}
& B_{\mathfrak{}}\left(\mathfrak{x}, \chi_{\mathfrak{x}}\left(i \partial_{\phi_{\mathfrak{x}}}\right)\right) e^{-i k_{\mathfrak{x}} \phi_{\mathfrak{x}}}=e^{-i k_{\mathfrak{x}} \phi_{\mathfrak{x}}} B_{\mathfrak{x}}\left(\mathfrak{x}, \chi_{\mathfrak{x}}\left(i \partial_{\phi_{\mathfrak{x}}}+k_{\mathfrak{x}}\right)\right) \text {. }
\end{aligned}
$$

If we choose a function $\chi\left(i \partial_{\phi}\right)$ such that $\chi\left(i \partial_{\phi}+k\right)=$ $f\left[\chi\left(i \partial_{\phi}\right)\right]$, then we have identified $a_{0} \rightarrow \chi\left(i \partial_{\phi}\right)$, and $a_{1}=f\left(a_{0}\right) \rightarrow f\left[\chi\left(i \partial_{\phi}\right)\right]=\chi\left(i \partial_{\phi}+k\right)$. Similar relations can 
be obtained for the $æ$-representations. From (77) we obtain then

$$
\begin{aligned}
B_{+} & \left(x, \chi\left(i \partial_{\phi}\right)\right) B_{-}\left(x, \chi\left(i \partial_{\phi}\right)\right) \\
\quad & B_{-}\left(x, \chi\left(i \partial_{\phi}+k\right)\right) B_{+}\left(x, \chi\left(i \partial_{\phi}+k\right)\right)=R\left[\chi\left(i \partial_{\phi}\right)\right], \\
B_{\mathfrak{x}+} & \left(\mathfrak{x}, \chi_{\mathfrak{x}}\left(i \partial_{\phi_{\mathfrak{x}}}\right)\right) B_{\mathfrak{x}-}\left(\mathfrak{x}, \chi\left(i \partial_{\phi_{\mathfrak{x}}}\right)\right) \\
& \quad-B_{\mathfrak{x}}\left(\mathfrak{x}, \chi_{\mathfrak{x}}\left(i \partial_{\phi_{\mathfrak{x}}}+k_{\mathfrak{x}}\right)\right) B_{\mathfrak{x}+}\left(\mathfrak{x}, \chi_{\mathfrak{x}}\left(i \partial_{\phi_{\mathfrak{x}}}+k_{\mathfrak{x}}\right)\right) \\
& =R_{\mathfrak{x}}\left[\chi_{\mathfrak{x}}\left(i \partial_{\phi_{\mathfrak{x}}}\right)\right] .
\end{aligned}
$$

Introducing the operators $J_{3}=-(i / k) \partial_{\phi}$ and $J_{\mathfrak{x} 3}=-\left(i / k_{\mathfrak{x}}\right) \partial_{\phi_{x}}$ and combining (79) and (82), we may arrive at a deformed Lie algebras as follows:

$$
\begin{aligned}
& {\left[J_{+}, J_{-}\right]=\left[J_{q^{+}}, J_{q^{-}}\right]-\left[J_{p+}, J_{p^{-}}\right]} \\
& =\xi\left(J_{3}\right)=\xi_{q}\left(J_{q 3}\right)-\xi_{p}\left(J_{p 3}\right),
\end{aligned}
$$

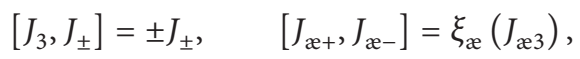

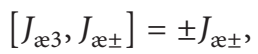

where $\xi\left(J_{3}\right) \equiv R\left[\chi\left(i \partial_{\phi}\right)\right]$ and $\xi_{\mathfrak{x}}\left(J_{\mathfrak{x} 3}\right) \equiv R_{\mathfrak{x}}\left[\chi_{\mathfrak{x}}\left(i \partial_{\phi_{\mathfrak{x}}}\right)\right]$ define the deformations. Different $\chi$ functions in (82) define different reparametrizations corresponding to several models. For example,

(1) the translational models $\left(a_{1}=a_{0}+k\right)$ correspond to $\chi(z)=z$. If $R$ is a linear function of $J_{3}$, the algebra becomes $\mathrm{SO}(2.1)$ or $\mathrm{SO}(3)$. Similar in many respects prediction is made in somewhat different method by Balantekin [17];

(2) the scaling models $\left(a_{1}=e^{k} a_{0}\right)$ correspond to $\chi(z)=$ $e^{z}$ and so forth.

6.2. The Unitary Representations of the Deformed Lie Algebra. In order to find the energy spectrum of the partner SUSY Hamiltonians

$$
\begin{aligned}
2 H_{-}\left(x, \chi\left(i \partial_{\phi}\right)\right)= & B_{-}\left(x, \chi\left(i \partial_{\phi}+k\right)\right) \\
\times & B_{+}\left(x, \chi\left(i \partial_{\phi}+k\right)\right), \\
2 H_{\mathfrak{x}}\left(æ, \chi_{\mathfrak{x}}\left(i \partial_{\phi_{\mathfrak{x}}}\right)\right)= & B_{\mathfrak{x}}\left(\mathfrak{x}, \chi_{\mathfrak{x}}\left(i \partial_{\phi_{\mathfrak{x}}}+k_{\mathfrak{x}}\right)\right) \\
& \times B_{\mathfrak{x}}\left(\mathfrak{x}, \chi_{\mathfrak{x}}\left(i \partial_{\phi_{\mathfrak{x}}}+k_{\mathfrak{x}}\right)\right),
\end{aligned}
$$

one must construct the unitary representations of deformed Lie algebra defined by (83) $[16,18]$. Using the standard technique, one defines up to additive constants the functions $g\left(J_{3}\right)$ and $g_{\mathfrak{x}}\left(J_{\mathfrak{3} 3}\right)$ as follows:

$$
\begin{aligned}
& \xi\left(J_{3}\right)=g\left(J_{3}\right)-g\left(J_{3}-1\right), \\
& \xi_{\mathfrak{x}}\left(J_{\mathfrak{x} 3}\right)=g_{\mathfrak{x}}\left(J_{\mathfrak{x} 3}\right)-g_{\mathfrak{x}}\left(J_{\mathfrak{x} 3}-1\right) .
\end{aligned}
$$

The Casimirs of this algebra can be written as $C_{2}=J_{-} J_{+}+$ $g\left(J_{3}\right)$ and accordingly $C_{\mathfrak{x} 2}=J_{\mathfrak{x}} J_{\mathfrak{x}}+g_{\mathfrak{x}}\left(J_{\mathfrak{x} 3}\right)$. In a basis in which $J_{3}$ and $C_{2}$ are diagonal, $J_{-}$and $J_{+}$are lowering and raising operators (the same holds for æ-representations). Operating on an arbitrary state $\mid h>$, they yield

$$
\begin{gathered}
J_{3}|h\rangle=h|h\rangle, \quad J_{-}|h\rangle=a(h)|h-1\rangle, \\
J_{+}|h\rangle=a^{*}(h+1)|h+1\rangle,
\end{gathered}
$$

where

$$
|a(h)|^{2}-|a(h+1)|^{2}=g(h)-g(h-1) .
$$

Similar arguments can be used for the operators $J_{(p, q) 3}$, $C_{æ 2}$, and $J_{æ \pm}$, which yield similar relations for the states $h_{\mathfrak{x}}$. The profile of $g(h)$ (and, thus, of $g_{\mathfrak{x}}\left(h_{\mathfrak{x}}\right)$ ) determines the dimension of the unitary representation. Having the representation of the algebra associated with a characteristic model, consequently we obtain the complete spectrum of the system. For example, without ever referring to underlying differential equation, we may obtain analytic expressions for the entire energy spectrum of extended Hamiltonian with self-similar potential. A scaling change of parameters is given as $a_{1}=Q a_{0}, a_{æ 1}=Q Q_{x} a_{\mathfrak{x} 0}$, at the simple choice $R\left(a_{0}\right)=-r_{1} a_{0}$, where $r_{1}$ is a constant. That is,

$$
\begin{aligned}
\xi\left(J_{3}\right) & \equiv-r_{1} \exp \left(-k J_{3}\right)=\xi_{q}\left(J_{q 3}\right)-\xi_{p}\left(J_{p 3}\right) \\
& =-r_{q 1} \exp \left(-k_{q} J_{q 3}\right)+r_{p 1} \exp \left(-k_{p} J_{p 3}\right),
\end{aligned}
$$

which yields

$$
\left[J_{+}, J_{-}\right]=\xi\left(J_{3}\right)=-r_{1} \exp \left(-k J_{3}\right), \quad\left[J_{3}, J_{ \pm}\right]= \pm J_{ \pm} .
$$

This is a deformation of the standard $\mathrm{SO}(2.1)$ Lie algebra, and therefore one gets

$$
\begin{aligned}
& g(h)=\frac{r_{1}}{e^{k}-1} e^{-k h}=-\frac{r_{1}}{1-Q} Q^{-h}, \quad Q=e^{k}, \\
& g_{\mathfrak{x}}\left(h_{\mathfrak{x}}\right)=\frac{r_{\mathfrak{x} 1}}{e^{k_{\mathfrak{x}}-1}} e^{-k_{\mathfrak{x}} h_{\mathfrak{x}}}=-\frac{r_{\mathfrak{x} 1}}{1-Q_{\mathfrak{x}}} Q_{\mathfrak{x}}^{-h_{\mathfrak{x}}}, \\
& Q_{æ}=e^{k_{x}} .
\end{aligned}
$$

For scaling problems [16] one has $0<q<1$, which leads to $k<0$. The unitary representation of this algebra for monotonically decreasing profile of the function $g(h)$, is infinite dimensional. Let the lowest weight state of the $J_{3}$ be $h_{\min }$, and then $a\left(h_{\min }\right)=0$. One can choose the coefficients $a(h)$ to be real. From (87), for an arbitrary $h=h_{\min }+n$, $n=0,1,2, \ldots$, we obtain

$$
a(h)^{2}=g(h-n-1)-g(h-1)=r_{1} \frac{Q^{n}-1}{Q-1} Q^{1-h} .
$$

The spectrum of the extended Hamiltonian $H_{-}\left(x, a_{1}\right)$ reads

$$
H_{-}|h\rangle=a(h)^{2}|h\rangle=r_{1} \frac{Q^{n}-1}{Q-1} Q^{1-h}|h\rangle,
$$


with the eigenenergies

$$
E_{n}(h)=r_{1} \alpha(h) \frac{Q^{n}-1}{Q-1}, \quad \alpha(h) \equiv Q^{1-h} .
$$

Similar expressions can be obtained for the $H_{æ-}$ and eigenenergies $E_{q n_{1}}$ and $E_{p n_{2}}\left(n \equiv\left(n_{1}, n_{2}\right), n_{1,2}=0,1,2, \ldots\right)$ as

$$
\begin{aligned}
& H_{q-}\left|h_{q}\right\rangle=a_{q}\left(h_{q}\right)^{2}\left|h_{q}\right\rangle=r_{q 1} \frac{Q_{q}^{n_{1}}-1}{Q_{q}-1} Q_{q}^{1-h_{q}}\left|h_{q}\right\rangle, \\
& H_{p-}\left|h_{p}\right\rangle=a_{p}\left(h_{p}\right)^{2}\left|h_{p}\right\rangle=r_{p 1} \frac{Q_{p}^{n_{2}}-1}{Q_{p}-1} Q_{p}^{1-h_{p}}\left|h_{p}\right\rangle,
\end{aligned}
$$

and that

$$
\begin{array}{r}
E_{q n_{1}}\left(h_{q}\right)=r_{q 1} \alpha_{q}\left(h_{q}\right) \frac{Q_{q}^{n_{1}}-1}{Q_{q}-1}, \\
\alpha_{q}\left(h_{q}\right) \equiv Q_{q}^{1-h_{q}}, \\
E_{p n_{2}}\left(h_{p}\right)=r_{p 1} \alpha_{p}\left(h_{p}\right) \frac{Q_{p}^{n_{2}}-1}{Q_{p}-1}, \\
\alpha_{p}\left(h_{p}\right) \equiv Q_{p}^{1-h_{p}} .
\end{array}
$$

Hence

$$
E_{n}(h)=E_{q n_{1}}\left(h_{q}\right)-E_{p n_{2}}\left(h_{p}\right)
$$

\section{Conclusions}

We addressed the classical analog of the extended phase space quantum mechanics of particle which have both bosonic and fermionic degrees of freedom, that is, the particle with odd degrees of freedom which gives rise to $(N=2)$-realization of the supersymmetry algebra. We obtain the integrals of motion. We use the iterative scheme to find the approximate ground state solutions to the extended Schrödinger-like equation and calculate the parameters which measure the breaking of extended SUSY such as the ground state energy. The approximation, which went into the derivation of solutions of (44), meets our interest that the ground state energy $\varepsilon$ is supposedly small. This gives direct evidence for the SUSY breaking. However, we calculate a more practical measure for the SUSY breaking, in particular in field theories which is the expectation value of an auxiliary field. We analyze in detail the nonperturbative mechanism for extended phase space SUSY breaking in the instanton picture and show that this has resulted from tunneling between the classical vacua of the theory. Finally, we present an analysis on the independent group theoretical methods with nonlinear extensions of Lie algebras from the extended phase space SUSY quantum mechanics. Using the factorization procedure we explore the algebraic property of shape invariance and spectrum generating algebra. Most of these Hamiltonians possess this feature and hence are solvable by an independent group theoretical method. We construct the unitary representations of the deformed Lie algebra.

\section{Acknowledgments}

The author would like to thank Y. Sobouti for drawing his attention to the extended phase space formulation of quantum mechanics. The knowledgable comments from the anonymous referees are much appreciated.

\section{References}

[1] Y. Sobouti and S. Nasiri, "A phase space formulation of quantum state functions," International Journal of Modern Physics B, vol. 7, no. 18, pp. 3255-3272, 1993.

[2] S. Nasiri, Y. Sobouti, and F. Taati, "Phase space quantum mechanics-direct," Journal of Mathematical Physics, vol. 47, no. 9, Article ID 092106, 15 pages, 2006.

[3] G. T. Ter-Kazarian and Y. Sobouti, "An extended phase-space stochastic quantization of constrained Hamiltonian systems," Journal of Physics A, vol. 41, no. 31, Article ID 315303, 8 pages, 2008.

[4] G. Ter-Kazarian, "An extended phase-space SUSY quantum mechanics," Journal of Physics A, vol. 42, no. 5, Article ID 055302, 12 pages, 2009.

[5] H. Nicolai, "Supersymmetry and spin systems," Journal of Physics A, vol. 9, no. 9, pp. 1497-1506, 1976.

[6] E. Witten, "Dynamical breaking of supersymmetry," Nuclear Physics B, vol. 188, no. 3, pp. 513-554, 1981.

[7] E. Witten, “Constraints on supersymmetry breaking," Nuclear Physics B, vol. 202, no. 2, pp. 253-316, 1982.

[8] A. Inomata and G. Junker, "Quasiclassical path-integral approach to supersymmetric quantum mechanics," Physical Review A, vol. 50, no. 5, pp. 3638-3649, 1994.

[9] A. Inomata, H. Kuratsuji, and C. C. Gerry, Path Integrals and Coherent States of $S U(2)$ and $S U(1,1)$, Singapore, 1992.

[10] J. Gamboa and M. Plyushchay, "Classical anomalies for spinning particles," Nuclear Physics B, vol. 512, no. 1-2, pp. 485-504, 1998.

[11] G. Grignani, M. Plyushchay, and P. Sodano, "A pseudoclassical model for $P, T$-invariant planar fermions," Nuclear Physics B, vol. 464, no. 1-2, pp. 189-212, 1996.

[12] J. L. Cortés, M. S. Plyushchay, and L. Velázquez, "A pseudoclassical model for the massive Dirac particle in $d$ dimensions," Physics Letters B, vol. 306, no. 1-2, pp. 34-40, 1993.

[13] P. Salomonson and J. W. van Holten, "Fermionic coordinates and supersymmetry in quantum mechanics," Nuclear Physics B, vol. 196, no. 3, pp. 509-531, 1982.

[14] A. M. Polyakov, "Quark confinement and topology of gauge theories," Nuclear Physics B, vol. 120, no. 3, pp. 429-458, 1977.

[15] L. Gendenshtein, "Derivation of exact spectra of the Schrdinger equation by means of supersymmetry," Pisma Zhurnal Eksperimental noi i Teoreticheskoi Fiziki, vol. 38, pp. 299-302, 1983.

[16] A. Gangopadhyaya, J. Mallow, and U. P. Sukhatme, "Shape invariance and its connection to potential algebra," in Supersymmetry and Integrable Models, vol. 502 of Lecture Notes in Physics, pp. 341-350, Springer, 1998.

[17] A. B. Balantekin, "Algebraic approach to shape invariance," Physical Review A, vol. 57, no. 6, pp. 4188-4191, 1998.

[18] F. Cooper, A. Khare, and U. Sukhatme, "Supersymmetry and quantum mechanics," Physics Reports, vol. 251, no. 5-6, pp. 267385, 1995. 


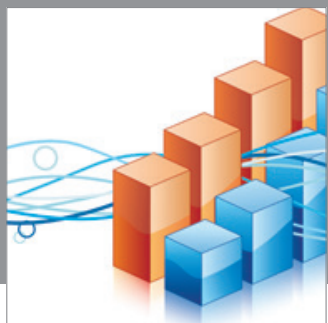

Advances in

Operations Research

mansans

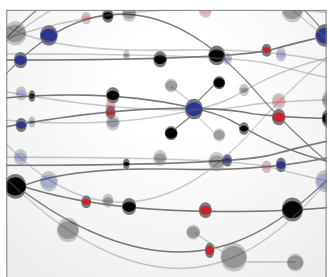

The Scientific World Journal
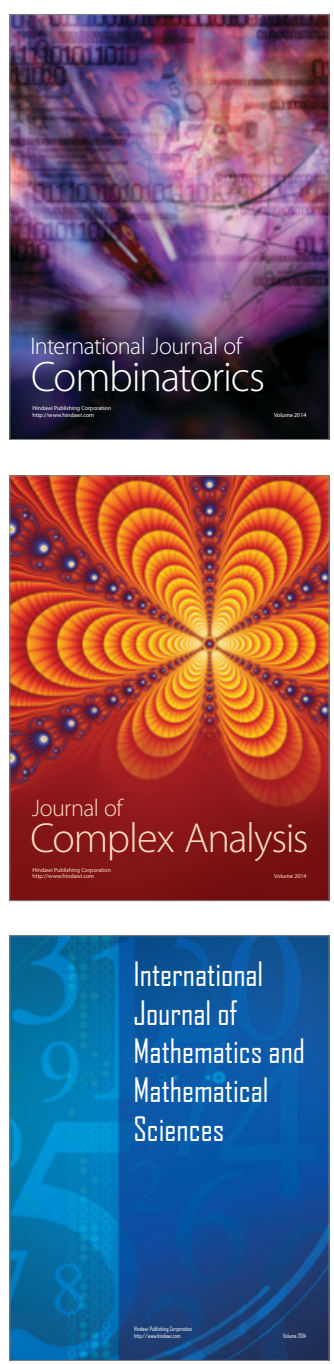
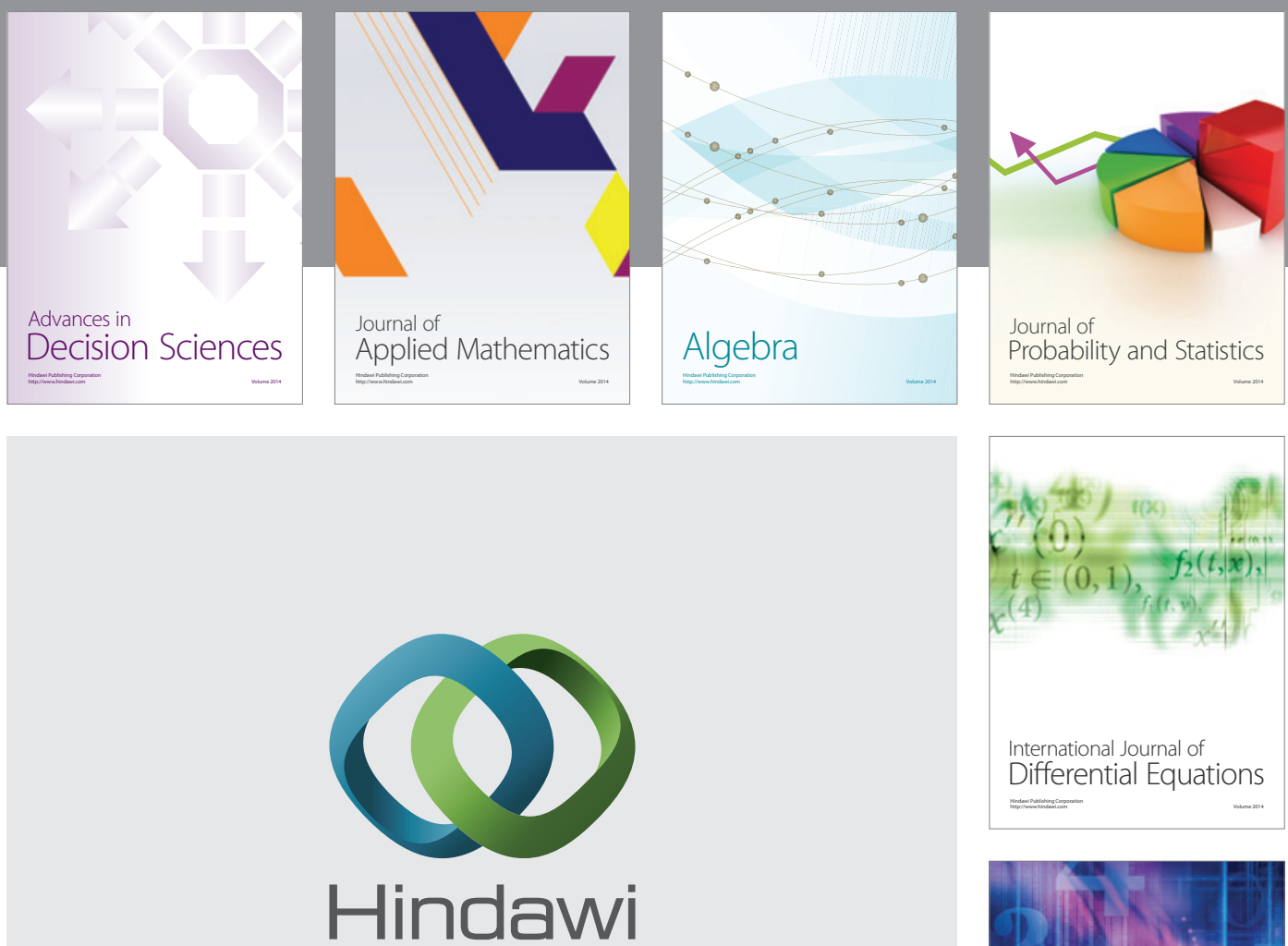

Submit your manuscripts at http://www.hindawi.com
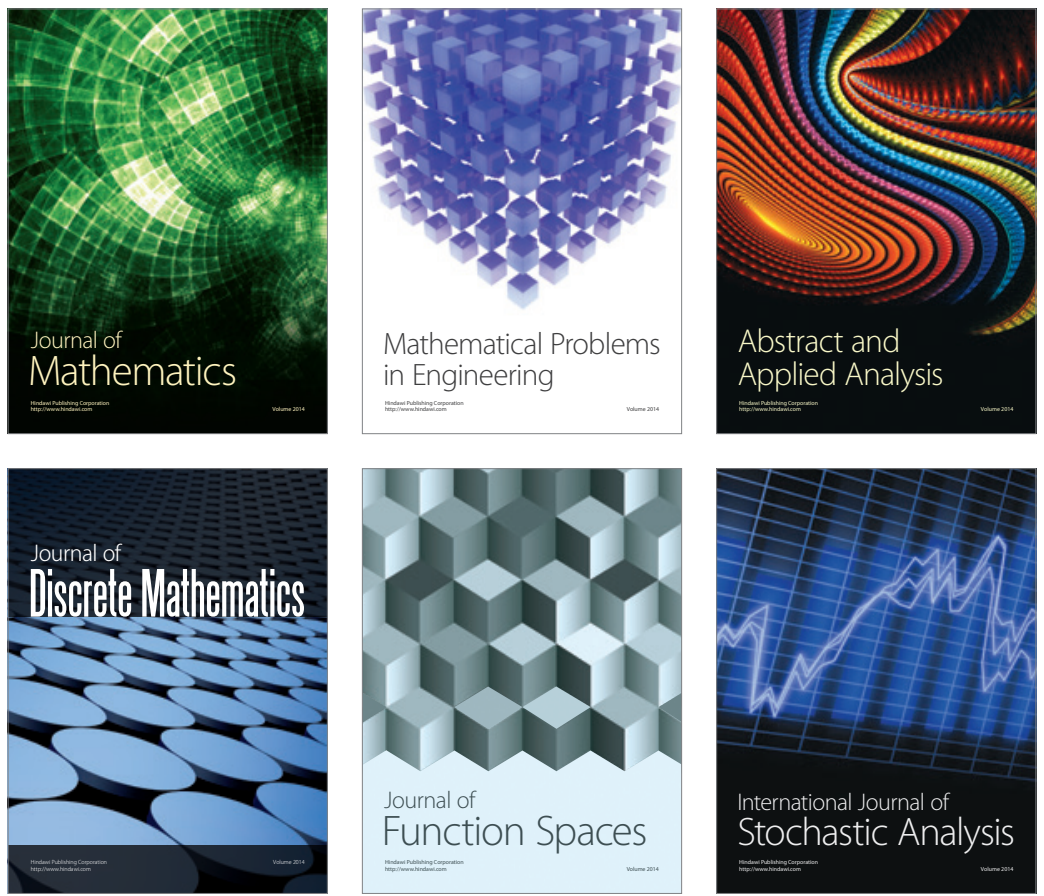

Journal of

Function Spaces

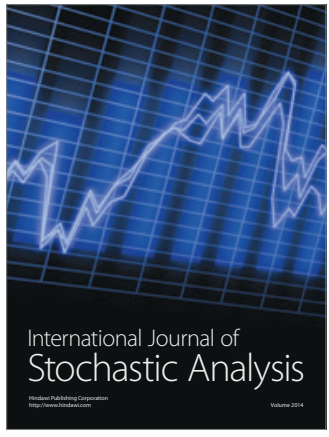

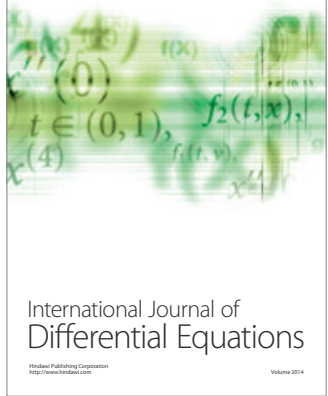
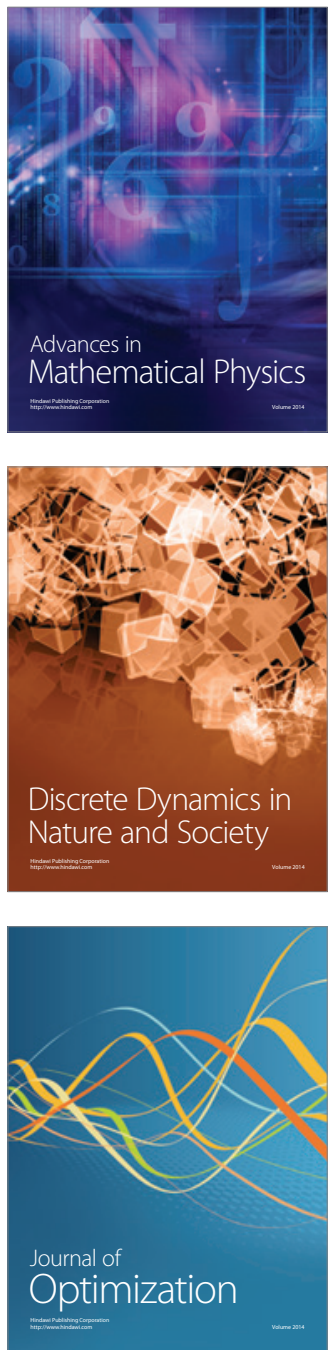